\title{
Generation of Squeezed States by Parametric Down Conversion
}

\author{
Ling-An Wu, H. J. Kimble, J. L. Hall, ${ }^{(a)}$ and Huifa Wu \\ Department of Physics, University of Texas at Austin, Austin, Texas 78712 \\ (Received 11 September 1986)
}

\begin{abstract}
Squeezed states of the electromagnetic field are generated by degenerate parametric down conversion in an optical cavity. Noise reductions greater than $50 \%$ relative to the vacuum noise level are observed in a balanced homodyne detector. A quantitative comparison with theory suggests that the observed squeezing results from a field that in the absence of linear attenuation would be squeezed by greater then tenfold.
\end{abstract}

PACS numbers: $42.50 . \mathrm{Dv}, 03.65-\mathrm{w}, 42.65 . \mathrm{Ky}$

While discussions of minimal-uncertainty states date to the earliest days of quantum mechanics, analyses dealing specificially with the electromagnetic field have been actively pursued for the past fifteen years. ${ }^{1,2}$ Particular attention has been focused on squeezed states of the field which are characterized by a phase-dependent redistribution of quantum fluctuations such that the dispersion in one of two quadrature components of the field is reduced below the level set by the symmetric distribution of the vacuum state. ${ }^{3}$ In terms of the familiar photon annihilation and creation operators $\left(\hat{a}, \hat{a}^{\dagger}\right)$ for a single-mode field of frequency $\omega_{1}$, a pair of conjugate quadrature operators is given by $\hat{X}_{+}=\left(\hat{a}+\hat{a}^{\dagger}\right)$ and $\hat{X}_{-}=-i\left(\hat{a}-\hat{a}^{\dagger}\right)$ with commutation relation $\left[\hat{X}_{+}, \hat{X}_{-}\right]$ $=2 i$ and corresponding uncertainty product $\Delta X_{+} \Delta X_{-}$ $\geq 1{ }^{4}$ The electric field operator $E$ is then expressed as $\hat{E}=E_{0}\left[\hat{X}+\cos \left(\omega_{1} t\right)+\hat{X}-\sin \left(\omega_{1} t\right)\right]$. Light in a coherent state (approximated by a single-mode laser) or in a vacuum state is in a minimal-uncertainty state $\left(\Delta X_{+} \Delta X_{-}=1\right)$ with equal variance for each of the two quadrature components. Squeezed states may or may not be minimal-uncertainty states but are such that one quadrature component has variance $\left(\Delta X_{ \pm}\right)^{2}<1$. The fluctuations expressed by the uncertainty product can be graphically represented [as shown in Fig. 1(a)] by a symmetric error circle for a coherent state (dashed line) while for a squeezed state this error circle is squeezed into an error ellipse (solid line). An analysis based on the Glauber-Sudarshan phase-space functional demonstrates that squeezed states of light lie outside the realm of any classical theory of the field. Since coherent laser beams of macroscopic intensity can be squeezed, one can argue that squeezed states represent a macroscopic quantum effect. It is the quantum nature of these states that gives rise to a host of intriguing possibilities associated with precision measurement at the quantum level and with the interaction of simple atomic systems with squeezed radiation.

In the past year squeezing has been observed in several laboratories, ${ }^{5-8}$ beginning with the landmark experiment of Slusher et al. ${ }^{5}$ While firmly establishing the existence of this phenomenon, these initial experiments ${ }^{5-7}$ demonstrated only modest reductions of $4-17 \%$ in noise power relative to the vacuum-state limit. In this Letter, we report experiments in which the recorded noise level in homodyne detection has been reduced by greater than
$50 \%$ relative to the level set by the vacuum state of the field. ${ }^{8}$ Furthermore, a quantitative comparison of our measurements with theoretical predictions leads to the

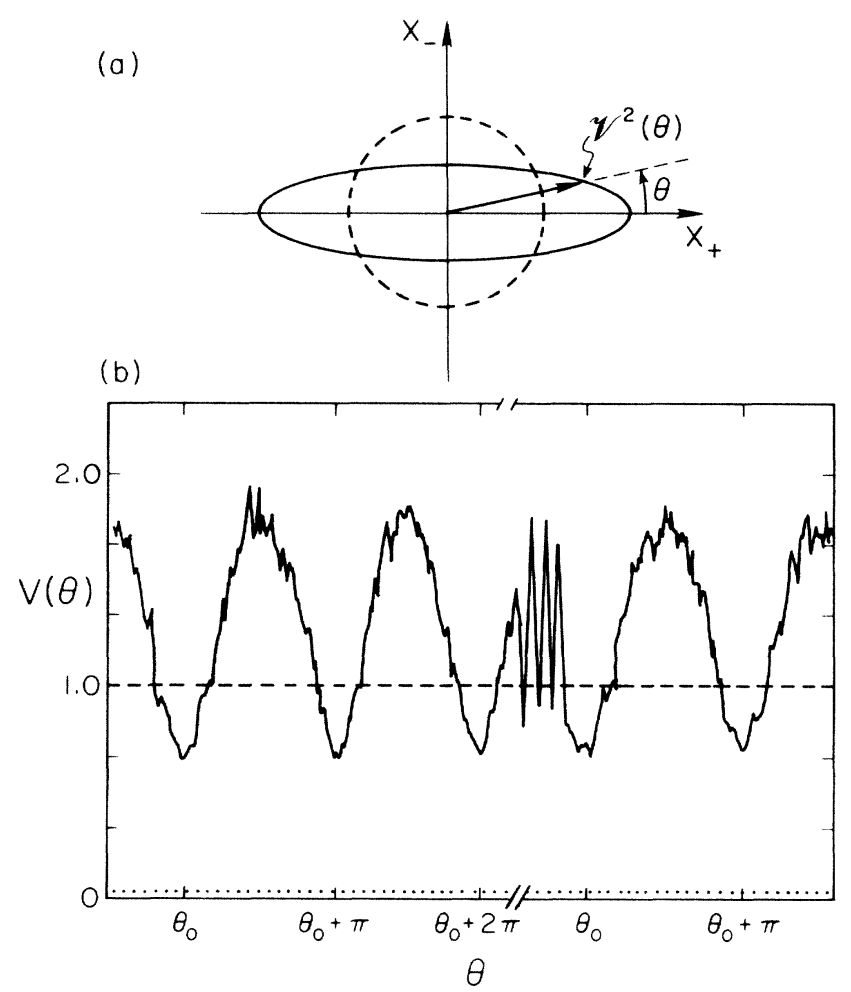

FIG. 1. (a) Phase plot of the uncertainties in the quadrature amplitudes of the electric field. The solid line represents the variance $v^{2}(\theta)$ of the field $\hat{X}(\theta)=\hat{X}+\cos \theta+\hat{X}-\sin \theta$ as a function of $\theta$ for a squeezed state; the dashed line is for the vacuum state. (b) Measurement of the phase dependence of the quantum fluctuations in a squeezed state produced by degenerate parametric down conversion. The plot corresponds roughly to the quantity $v(\theta)$ as in (a). More precisely, the phase dependence of the rms noise voltage $V(\theta)$ from a balanced homodyne detector is displayed as a function of local oscillator phase $\theta$ at fixed analysis frequency $(1.8 \mathrm{MHz})$ and bandwidth $(100$ $\mathrm{kHz}$ ) in the spectral distribution of photocurrent fluctuations. With the OPO input blocked, the vacuum field entering the signal port of the detector produces the noise voltage given by the dashed line with no sensitivity on $\theta$. With the OPO input present, the dips below the vacuum level represent a $50 \%$ reduction in noise power relative to the vacuum noise level. Note that the ordinate is a linear scale in noise voltage. The dotted line is the amplifier noise level. 
conclusion that the observed noise reduction resulted from a field that would exhibit more than tenfold squeezing in the absence of avoidable losses present in the current experiment. Finally, as expected theoretically for the type of squeezing process utilized, our measurements indicate that the field state is not only a squeezed state but is a minimal-uncertainty state as well.

In contrast to other research in this area that has investigated four-wave mixing for squeezed-state generation, our experiments involve the process of degenerate parametric down conversion in which photons of frequency $\omega_{2}$ are converted into correlated pairs of photons near the frequency $\omega_{1}=\omega_{2} / 2$. The subharmonic field is produced in a squeezed state. A diagram of our experimental arrangement is shown in Fig. 2. The down conversion occurs in the cavity $\left(\mathrm{M}, \mathrm{M}^{\prime}\right)$ of length $100 \mathrm{~mm}$ containing a nonlinear-optical crystal of $\mathrm{MgO}: \mathrm{LiNbO}_{3}$ of dimension $25 \times 9 \times 8 \mathrm{~mm}$ heated to the phase-matching temperature of $98^{\circ} \mathrm{C}$. The pump $P_{2}$ at $\omega_{2}$ for the optical parametric oscillator ${ }^{9}$ (OPO) is obtained by frequency doubling from 1.06 to $0.53 \mu \mathrm{m}$ with a crystal of $\mathrm{Ba}_{2} \mathrm{NaNb}_{5} \mathrm{O}_{15}$ inside the cavity of a frequency-stabilized neodymium-doped yttrium aluminum garnet laser. ${ }^{10}$ The field at $\omega_{2}$ enters the OPO cavity through the mirror $M$ with transmission coefficient of $3.5 \%$ at $0.53 \mu \mathrm{m}$ and $0.06 \%$ at $1.06 \mu \mathrm{m}$. $M^{\prime}$ is coated for low transmission at $0.53 \mu \mathrm{m}$ and for either $4.3 \%$ or $7.3 \%$ transmission at $1.06 \mu \mathrm{m}$. The lithium niobate crystal has dual-band antireflection coatings to minimize loss at 0.53 and 1.06 $\mu \mathrm{m}$ resulting in transmission at $\omega_{1}$ of $98 \%-99 \%$. A fraction of the downwardly converted light from the interac-

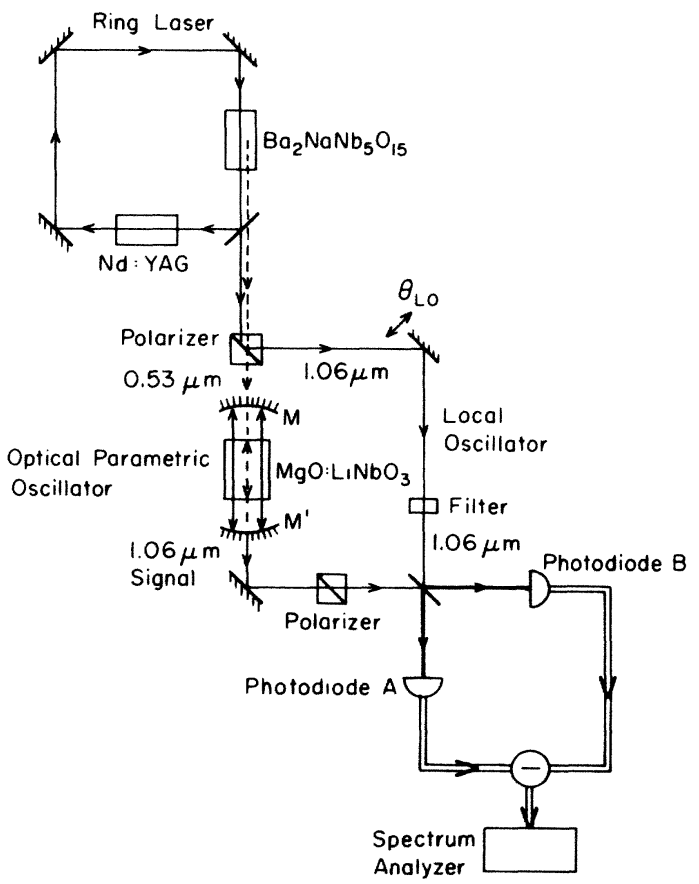

FIG. 2. Diagram of the principal elements of the apparatus for squeezed-state generation by degenerate parametric down conversion. tion in the OPO exits through $\mathbf{M}^{\prime}$ and is combined with the original laser emission, which acts as a strong local oscillator beam $(P \approx 1 \mathrm{~mW})$, in a balanced homodyne detector $^{11}$ formed by two InGaAs photodiodes DA and DB of quantum efficiency $\eta=0.90 \pm 0.04$ and associated amplifiers. The filter and polarizer ensure that only light of frequency $\omega_{1}$ enters the detector.

The length of the OPO cavity is servo controlled to lock a longitudinal-mode resonance to the frequency of the incident green beam at $0.53 \mu \mathrm{m}$ with an rf sideband technique. ${ }^{12}$ With the cavity stabilized in this fashion, the point of degenerate operation is identified by a search in temperature that tunes the birefringence of the crystal to bring successive longitudinal modes at $1.06 \mu \mathrm{m}$ into simultaneous resonance with the strong intracavity field at $0.53 \mu \mathrm{m}$. An auxilliary beam at $1.06 \mu \mathrm{m}$ can be injected into the cavity through $M$ to facilitate this search. When frequency offset with an acousto-optic modulator, this injected beam also allows optimization of the heterodyne efficiency $\varepsilon=0.90 \pm 0.10 .^{13}$ This injected beam is blocked for all subsequent measurements.

The observation of squeezing of the signal beam centers on an analysis of the spectral distribution of fluctuations of the signal formed by the subtraction of the two photocurrents $\left(I_{A}, I_{B}\right)$ produced by photodiodes (DA,DB). Figures 1(b) and (3) display the phase dependence of the rms noise voltage $V(\theta)$ from the balanced homodyne receiver as a function of local oscillator phase $\theta$ at fixed analysis frequency. The dashed line corresponds to the noise level $V_{0}$ set by vacuum fluctuations and is obtained either by blocking the signal input or equivalently by tuning the temperature of the lithium niobate crystal to shift the cavity resonance at $\omega_{1}$ from coincidence with the locked resonance at $\omega_{2}$. That this is indeed the vacuum noise level is demonstrated by the use of well-characterized hybrid junctions to combine the photocurrents $\left(I_{A}, I_{B}\right)$ with either $0^{\circ}$ or $180^{\circ}$ phase difference with the OPO input blocked. Since in the $0^{\circ}$

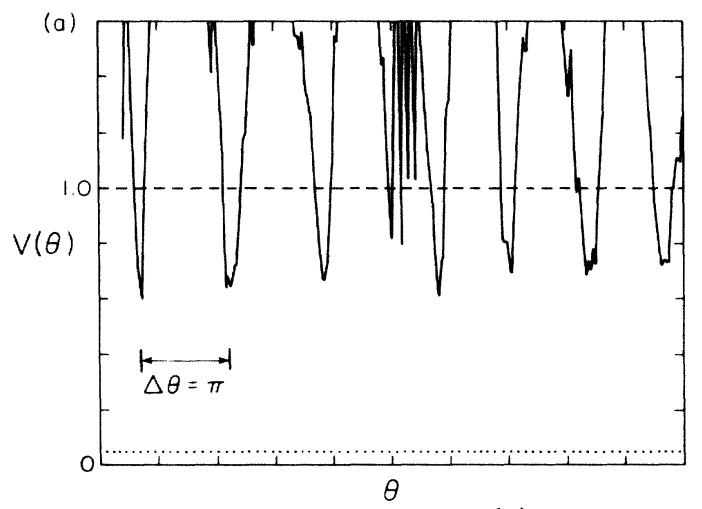

FIG. 3. Dependence of noise voltage $V(\theta)$ on local oscillator phase $\theta$ for the signal field produced by the subthreshold OPO. The scale has been expanded relative to Fig. 1(b) to display the deviations below the vacuum noise level (dashed line) more clearly. Otherwise the notation is as in Fig. 1(b). $V_{0}=250 \mu \mathrm{V}$; detection bandwidth $=100 \mathrm{kHz} ; v / 2 \pi=1.2 \mathrm{MHz}$. 
case excess local oscillator fluctuations add, while in the $180^{\circ}$ case they are suppressed, this measurement allows us to deduce that the laser amplitude fluctuations are at the "shot-noise" level to within $\pm 2 \%$ for spectral frequencies greater than $1 \mathrm{MHz}$. Furthermore, for the $180^{\circ}$ phase difference employed in the squeezing experiments, amplitude modulation on the local oscillator beam is suppressed by greater than $20 \mathrm{~dB}$ by the balanced detector arrangement. Measurements with equivalent illumination from a spectrally filtered incandescent lamp confirm that the noise spectra of the lamp and of the laser coincide to within $\pm 5 \%$ for the same dc photocurrents. In Figs. 1(b) and 3 the time taken for the entire sweeps is $0.2 \mathrm{~s}$; the sharp features in each of the traces correspond to the flyback of the piezoelectric crystal used to scan the local oscillator phase.

Values of $V(\theta)$ below the dashed lines in Figs. 1(b) and 3 represent observations of squeezing of the incident signal field. We characterize the degree of squeezing by introducing the variable $R(\theta)$ defined by

$$
\left.R(\theta)=\left[V^{2}(\theta) V_{A}^{2}\right)\right] /\left(V_{0}^{2}-V_{A}^{2}\right),
$$

which gives the reduction $R=R\left(\theta_{-}\right)$or enhancement $R_{+}=R\left(\theta_{+}\right)$in the detected noise power relative to the vacuum level. In $(1), V(\theta)$ is the rms noise voltage with $\theta_{-}\left(\theta_{+}\right)$denoting the position of the minimum (maximum) voltage, $V_{0}$ is the voltage corresponding to the vacuum level and $V_{A}$ is the level of amplifier noise. From an analysis of a large number of traces under the separate sets of operating conditions represented by Figs. 1 (b) and 3, we estimate noise reduction factors $R$ - of $0.47 \pm 0.02$ and $0.41 \pm 0.05$, respectively. Although Fig. 3 is a trace from a data set of our best measurements in terms of the degree of squeezing achieved [resulting from a favorable value of $g$ as described in Eq. (3)], we can reproducibly achieve $R_{-}<0.50$, which represents observed squeezing by more than a factor of 2 . We stress that this result involves no corrections for detector efficiency or for losses suffered by the signal beam in propagation to the detector.

To make a quantitative comparison of our results with theoretical predictions, we must consider the relationship of the detected external field to the intracavity field of the OPO. ${ }^{14,15}$ In a rotating frame of frequency $\omega_{1}$, we denote the annihilation and creation operators for the intracavity field by $\left(\hat{a}(t), \hat{a}^{\dagger}(t)\right)$ and introduce the quadrature amplitude $\hat{X}_{\theta}(t)=e^{-i \theta} \hat{a}(t)+e^{i \theta} \hat{a}^{\dagger}(t)$. The spectrum of squeezing $S(v, \theta)$ of the output field assuming an ideal single-ended cavity of damping rate $\Gamma_{1}$ is then given by ${ }^{15,16}$

$$
S(v, \theta)=2 \Gamma_{1} \int_{-\infty}^{+\infty} d \tau\left\langle T: \hat{X}_{\theta}(t), \hat{X}_{\theta}(t+\tau):\right\rangle e^{-i v \tau},
$$

where the colons denote normal ordering, $T$ stands for time ordering, and the covariance $\langle a, b\rangle=\langle a b\rangle-\langle a\rangle\langle b\rangle$. If the cavity is not a single-port device, the observable phase-dependent variations in the amplitude of the output field are degraded by the factor $(1+g)^{-1}$, where $g$ expresses the ratio of loss rate $\gamma_{1}^{\prime}$ by all avenues other than through the output coupler ( $\mathrm{M}^{\prime}$ in Fig. 2) to the loss rate $\gamma_{1}$ through the output coupler $\left(\Gamma_{1}=\gamma_{1}+\gamma_{1}^{\prime}\right)$. The ratio $R$ of Eq. (1) obtained from an analysis of the spectral distribution of current fluctuations from the balanced homodyne detector can be related to $S$ following the treatments of Shapiro ${ }^{17}$ and Mandel, ${ }^{18}$

$$
R(\Omega, \theta)=1+[\alpha /(1+g)] S(\Omega, \theta),
$$

where $\Omega=v / \Gamma_{1}$ and the phase angle $\theta$ is set by the local oscillator phase. Exclusive of the beam splitter, $\alpha$ expresses the detection quantum efficiency for propagation from the output mirror $\mathbf{M}^{\prime}$ to either photodiode, with inclusion of the quantum efficiency $\eta$ of the detector and the homodyne efficiency $\varepsilon$. Rearranging Eq. (3) to express $S$ in terms of $R$ and with the further association of $R \pm$ with $S_{ \pm}$, we are thus able to extract the spectrum of squeezing from measurements such as those in Figs. 1 (b) and 3.

Figure 4 shows the dependence of $S_{-}=S\left(\Omega, \theta_{-}\right)$on pump ratio $r=P_{2} / P_{0}$, with $P_{0}$ the incident power at $\omega_{2}$ required to reach the threshold for parametric oscillation. For the data of Fig. $4, \alpha=0.74 \pm 0.08$ and $(1+g)^{-1}=0.70 \pm 0.07$. The full curve is the theoretical prediction from Ref. 16, Eq. (4.4). We stress that the comparison of theory and experiment is made with no adjustable parameters and no fitting has been attempted. A further contact with theory is obtained by exploring the dependence of $S_{-}(\Omega)$ on $\Omega$. For a fixed value $r=0.55$ and with the measured values of $(1+g), \alpha$, and $\Gamma_{1}$, we have confirmed that the diminution of $\left|S_{-}(\Omega)\right|$ with increasing $\Omega$ is in reasonable agreement with theoretical prediction. ${ }^{16}$

While attention has centered on the quantities $R_{-}, S_{-}$ that express the degree of squeezing of the field in terms

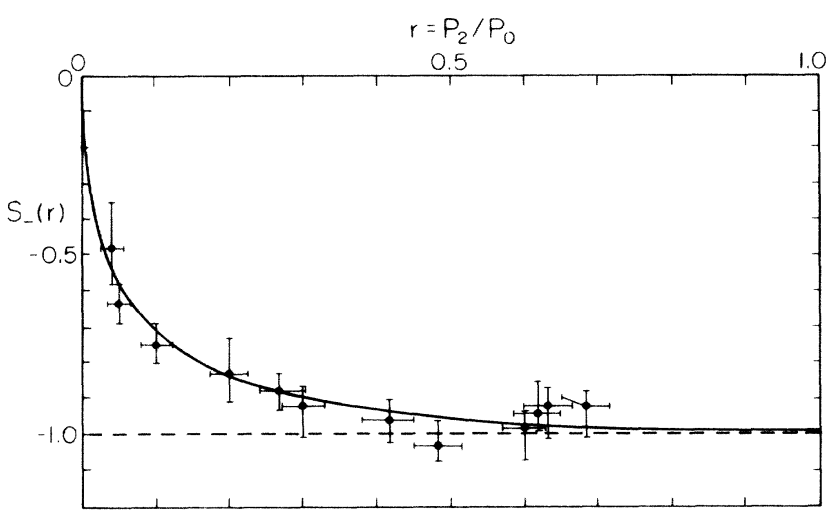

FIG. 4. Comparison of the values $S_{-}(r)$ derived from the measurements with those given by theory (solid curve) for fixed $\Omega=0.21$ with the measured values $\alpha /(1+g)=0.52$ and $P_{0}=30 \mathrm{~mW}$. Perfect squeezing occurs for $S_{-}(\Omega)=-1$ and is indicated by the dashed line. The uncertainties shown for each point derive from uncertainties in the determination of $R$ - and of $P_{2}$ at a given operating point and do not include the overall uncertainties in $\alpha, g$, or $P_{0}$. 
of noise reduction, we can examine as well the enhancement in fluctuations in the conjugate field quadrature expressed by $R_{+}, S_{+}$and shown in Fig. 1. If the field produced by the OPO is in a minimal-uncertainty state in the sense that

$$
M \equiv\left[1+S_{-}(\Omega)\right]\left[1+S_{+}(\Omega)\right]=1
$$

as is predicted from an examination of the results in Ref. 16 , then there should be a definite relationship between the minimum and maximum noise levels recorded in our experiments. While the current uncertainties in the values of $R, \alpha$, and $g$ translate into a large uncertainty for the product $M$ when Eqs. (3) and (4) are combined, we find that $M$ lies within the range $0.8<M<1.2$ for data examined over the range $1.4<S_{+}<8.1$. This observation, together with the agreement between theory and experiment evidenced in Fig. 4, lends support to the inference that the field state is a minimal uncertainty state before degradation by the various loss mechanisms.

In summary, we have employed the process of parametric down conversion in a subthreshold optical parametric oscillator to reduce the noise level in homodyne detection by more than a factor of 2 relative to the vacuum noise level. The spectrum of squeezing extracted from our measurements (Fig. 4) indicates that the observed squeezing resulted from a field squeezed more than tenfold. Straightforward improvements in the cavity design and in the detection efficiencies should translate this large degree of squeezing into a comparably large reduction in the observed noise level in our balanced homodyne detector. Beyond the demonstration of the feasibility of achievement of a large degree of squeezing, our work should have direct application to a number of exciting problems in optical physics involving the nonclassical nature of squeezed radiation.

This research was supported by the Venture Research Unit of British Petroleum North America. The work at the Joint Institute for Laboratory Astrophysics, National Bureau of Standards and University of Colorado, Boulder, Colorado, was supported by the National Bureau of Standards, the U. S. Office of Naval Research, and the National Science Foundation. The technical contributions of T. L. Boyd and Min Xiao are gratefully acknowledged. The lithium niobate crystal was generously provided by Crystal Technology and the barium sodium niobate crystal by the Shanghai Institute of Ceramics, Academia Sinica. (a) Permanent address: Joint Institute for Laboratory Astrophysics, National Bureau of Standards and University of Colorado, Boulder, CO 80309.

1D. Stoler, Phys. Rev. D 1, 3217 (1970), and D 4, 1925 (1971).

${ }^{2}$ H. P. Yuen, Phys. Rev. A 13, 2226 (1976).

${ }^{3}$ For a review, see D. F. Walls, Nature (London) 306, 141 (1983).

${ }^{4}$ We denote Hilbert-space operators with a caret.

${ }^{5}$ R. E. Slusher, L. W. Hollberg, B. Yurke, J. C. Mertz, and J. F. Valley, Phys. Rev. Lett. 55, 2409 (1985); R. E. Slusher, B. Yurke, J. R. Klauder, and S. L. McCall, in Proceedings of the Joint Meetings: Fourteenth International Conference on Quantum Electronics and Sixth Annual Conference on Lasers and Electro-Optics, San Francisco, California, 9-13 June 1986 (to be published).

${ }^{6}$ R. M. Shelby, M. D. Levenson, S. H. Perlmutter, R. G. DeVoe, and D. F. Walls, Phys. Rev. Lett. 57, 691 (1986), and in Proceedings of the Joint Meetings: Fourteenth International Conference on Quantum Electronics and Sixth Annual Conference on Lasers and Electro-Optics, San Francisco, California, 9-13 June 1986 (to be published).

${ }^{7}$ M. W. Maede, P. Kumar, and J. H. Shapiro, in Proceedings of the Joint Meetings: Fourteenth International Conference on Quantum Electronics and Sixth Annual Conference on Lasers and Electro-Optics, San Francisco, California, 9-13 June 1986 (to be published).

${ }^{8} \mathrm{H}$. J. Kimble and J. L. Hall, in Proceedings of the Joint Meetings: Fourteenth International Conference on Quantum Electronics and Sixth Annual Conference on Lasers and Electro-Optics, San Francisco, California, 9-13 June 1986 (to be published).

${ }^{9}$ R. G. Smith, in Lasers: A Series of Advances, edited by A. K. Levine and A. DeMaria (Dekker and Marcel, New York, 1976), Vol. 4.

${ }^{10}$ K.-C. Peng, L.-A. Wu, and H. J. Kimble, Appl. Opt. 24, 938 (1985).

${ }^{11}$ H. P. Yuen and V. W. S. Chan, Opt. Lett. 8, 177 (1983).

${ }^{12}$ R. W. P. Drever, J. L. Hall, F. V. Kowalski, J. Hough, G. M. Ford, A. J. Munley, and H. Ward, Appl. Phys. B 31, 97 (1983).

${ }^{13}$ L. Mandel and E. Wolf, J. Opt. Soc. Am. 65, 413 (1975).

${ }^{14}$ B. Yurke and J. S. Denker, Phys. Rev. A 29, 1419 (1984).

${ }^{15}$ M. J. Collett and C. W. Gardiner, Phys. Rev. A 31, 3761 (1985).

${ }^{16}$ M. J. Collett and D. F. Walls, Phys. Rev. A 32, 2887 (1985).

17J. H. Shapiro, IEEE J. Quantum Electron. 21, 237 (1985).

${ }^{18} \mathrm{~L}$. Mandel, in Optics in Four Dimensions - 1980, edited by M. A. Machado and L. M. Narducci, AIP Conference Proceedings No. 65 (American Institute of Physics, New York, 1981). 